ÉGYPTE monde arabe

\section{Égypte/Monde arabe}

30-31 | 1997

Les visions de l'Occident dans le monde arabe

\title{
La Bosnie, la Umma, l'Occident : perceptions égyptiennes
}

\section{Iman Farag}

\section{(2) OpenEdition}

1 Journals

Édition électronique

URL : https://journals.openedition.org/ema/1644

DOI : 10.4000/ema.1644

ISSN : 2090-7273

\section{Éditeur}

CEDEJ - Centre d'études et de documentation économiques juridiques et sociales

\section{Édition imprimée}

Date de publication : 30 septembre 1997

Pagination : 185-210

ISSN : 1110-5097

\section{Référence électronique}

Iman Farag, «La Bosnie, la Umma, l'Occident : perceptions égyptiennes », Égypte/Monde arabe [En ligne], 30-31 | 1997, mis en ligne le 08 juillet 2008, consulté le 07 juillet 2022. URL : http:// journals.openedition.org/ema/1644; DOI : https://doi.org/10.4000/ema.1644

Ce document a été généré automatiquement le 7 juillet 2022.

Tous droits réservés 


\title{
La Bosnie, la Umma, l'Occident : perceptions égyptiennes
}

\author{
Iman Farag
}

\footnotetext{
« Tout ce qu'on peut classer est périssable. Ne dure que ce qui est susceptible de plusieurs interprétations. " Cioran, Aveux et anathèmes.
}

1 Lors de l'hiver 1994, particulièrement meurtrier dans la ville de Mallawi, théâtre d'affrontements violents entre islamistes et forces de l'ordre, un journaliste rapportait cette phrase : «Ici, c'est pire que la Bosnie ». Au-delà de la comparaison qui portait sur la violence proprement dite, les propos des habitants de Mallawi semblaient suggérer un lien entre ce qu'ils vivaient et ce qui se passait à l'autre bout du monde, dans une région dont ils venaient à peine d'apprendre le nom et dont ils ignoraient au demeurant l'emplacement sur la carte. Entre autres actes de violence, un homme avait abattu de sang froid et sans raison apparente quatre coptes. La rumeur veut que la veille, il ait regardé les scènes de massacre en Bosnie à la télévision.

2 Peu importe que ce propos soit ou non fondé ; le fait même qu'il acquière le statut de rumeur signifie qu'il puisse être - même si ce n'est pas le cas aux yeux de tous - une éventualité parfaitement plausible. Il n'y a pas beaucoup de sens à rechercher une explication du côté de l'impact des médias, pas plus qu'il n'y en aurait à présenter cet "acte isolé » comme un épiphénomène sa'îdî propre à la Haute-Égypte - la gâchette facile - ou à l'expliquer par l'ampleur des campagnes pro-bosniaques menées dans les mosquées de la ville, même si l'on sait qu'ici les prônes sont plus virulents et plus présents dans l'espace urbain que dans d'autres villes. Ces facteurs ne permettent que d'expliquer et dans une certaine mesure seulement, le «comment». S'agissant du " pourquoi ", il est généralement donné en termes de solidarité islamique. Sans nier ce que l'évocation de ce lien aurait d'efficace, il semble nécessaire d'examiner les modalités de cette efficacité et ses multiples mises en œuvre dans dés situations particulières. Autrement dit, il s'agit, à travers les perceptions égyptiennes de la question bosniaque, d'interroger l'actualisation et l'activation d'une représentation. 
Objectivées par les intéressés en termes de Umma, ces perceptions permettent un retour sur la «catégorie » et sur ses usages sociaux et nationaux multiples, voire contradictoires. Ce qui constitue la seule manière d'échapper à la lecture substantialiste qui voit dans le lien de solidarité islamique une essence immuable et dans la Umma, communauté des croyants musulmans, une explication nécessaire et suffisante. Interroger la catégorie Umma au travers des perceptions égyptiennes de la Bosnie, c'est, simultanément, questionner les divisions du monde que produisent ces perceptions.

\section{Constructions autour de la Umma}

4 Pour l'insider, la Umma se passe de justification. Elle est le territoire actuel de l'islam mais aussi sa mémoire et l'un comme l'autre sont balisés par un lien de solidarité qui, s'il n'implique pas de rupture avec l'universel ou avec la contemporanéité, n'en est pas moins un lien spécifique. À l'appui, ce hadîth dans lequel l'empathie entre croyants fait d'eux un seul et même corps : si l'une des parties venait à se plaindre d'un mal, c'est le tout qui répondrait à son appel. Que Mallawi se sente en phase avec Sarajevo, cela est dans l'ordre des choses.

5 Pour l'observateur de bonne foi, il y aurait quelque chose d'aberrant dans cette forme de solidarité qui ne s'énonce ni en termes politiques, ni en termes humanitaires, mais bien en termes religieux. Parler de "chrétienté » cela aurait-il encore un sens quelconque ? Cependant, en faisant appel, tant à l'arsenal islamologique, qu'aux lieux communs du discours de ou sur la réislamisation, la Umma va de soi également. Elle s'inscrirait dans le dogme musulman, dans la genèse historique d'une communauté des croyants, ou encore dans le vécu de sociétés plus musulmanes que les sociétés chrétiennes, par exemple, ne seraient chrétiennes - sociétés musulmanes qui de plus vivraient un moment de réislamisation. La Umma serait en quelque sorte le signe d'une sécularisation inachevée, d'un déficit de légitimité des États-nations, d'une configuration qui confirmerait l'absence de dissociation entre cosmologie et histoire, caractéristique propre aux « communautés imaginaires » territorialisées, nationales et modernes (Anderson, 1983). Une Umma amenée à disparaître si l'histoire suivait son cours "normal» mais remise au goût du jour, puisque les sociétés musulmanes contemporaines vivent précisément des phénomènes de «retours ». Culturalisme et évolutionnisme se retrouvent ici sur un même terrain.

6 Pour peu qu'il soit de mauvaise foi, l'observateur pourra reprendre certains des propos énoncés lors de la guerre du Golfe, et qui font de la Umma, en partant d'un mauvais jeu de mots en apparence étymologique, « un fantasme ombilical», "l'aspiration à une seule et même origine symbolique, sans immixtion de l'autre » (Sibony, 1991). La Umma serait ainsi l'une de ces fatalités qui frappent certaines parties de l'humanité, lesquelles ne pourraient en sortir qu'au prix de leur propre négation. Dans de tels propos, la Umma devient hyperbole, fratrie même, d'une société sans individus : «ils » relèvent d'appartenances, mais leurs appartenances ne peuvent leur appartenir.

7 Ces trois positions ne sont pas aussi contradictoires qu'elles en ont l'air : la Umma, ultime expression de la solidarité musulmane serait ainsi une donnée qui s'inscrirait dans l'histoire, la géographie, ou la "psychologie des foules». Dans les faits, que la Umma et ses diverses manifestations soient considérées comme légitimes ou non, il y a 
reconnaissance d'une donnée, de son caractère naturel et en quelque sorte, accord sur les termes du désaccord.

C'est d'une proposition inverse que l'on partira. La Umma comme élément structurant d'une vision $\mathrm{du}$ monde proprement dite est une construction réactualisée, aux frontières mouvantes : elle n'acquiert sa "matérialité » qu'au travers des énoncés de ceux qui - insiders mais aussi outsiders - s'y reconnaissent ou la reconnaissent ; c'est une catégorie issue des capacités d'induction d'acteurs qui la produisent, l'instrumentalisent en même temps qu'elle leur échappe. Peu nous importe à cet égard qu'à l'instar d'autres catégories d'identification, «sa mythologie soit plus lourde que son anthropologie » (Ben Achour, 1991).

Par la manière dont elle se le représente, la Umma participe de l'ordre mondial autant qu'elle en porte les marques. On voit mal comment cette catégorie serait à l'abri de certains effets: ainsi, à l'heure où l'on évoque l'«Internationale islamique », c'est «l'internationalisme » qui tombe en désuétude avec la fin du monde bipolaire et la mise en place de dynamiques transgressant les frontières nationales (culturelles, écologiques, humanitaires, mais aussi de migrations économiques) - dynamiques qui peuvent donner lieu à de nouvelles solidarités comme elles peuvent d'ailleurs générer de nouvelles tensions. Internationalisme ou trans-nationalisme - ce qui n'est pas la même chose - la Umma comme lecture de la carte du monde se redéfinit également face à l'invention de nouvelles visées nationalitaires et alors même que des cadres nationaux auraient tendance à paraitre comme vides.

D'aucuns diraient que l'ordre mondial se caractérise par la tension croissante entre les « appels » identitaire, utilitaire et citoyen (Badie, 1996). En tant que telle, la Umma n'est pas fermée à ces transformations : elle se redéfinit face à une série de mutations dans les représentations dont elle fait partie. Que les sociétés musulmanes soient par définition plus vulnérables que d'autres à l'un ou l'autre de ces appels constitue précisément la prémisse de nombres de lectures, et qu'il nous faut rejeter explicitement dès le départ.

11 Du simple fait de leur " islamité », les interactions entre les membres de ce même corps représenté qu'est la Umma, seraient-elles irréductibles dans leur spécificité par rapport à celles qui structurent de manière générale "la vision de l'Autre »? Seraient-elles soumises comme partout ailleurs aux effets d'échelle, de proximité et de hiérarchie, autrement dit de centre(s) et de périphéries? À la superposition d'une "carte légendaire » des lieux symboliques et d'une « cartographie réelle » des points chauds ? Les perceptions respectives sont-elles marquées et par l'auto-définition et par ce qui précisément provient du dehors de cette Umma et contribue à en redéfinir constamment les frontières? Il s'agira ici de montrer comment la saisie d'une question exprimée en termes de Umma -la cause bosniaque - opère à partir du "terroir " égyptien en même temps qu'elle met en œuvre les multiples registres de perception de l'Ailleurs, la Umma pouvant être définie précisément comme un registre parmi d'autres.

12 Le débat sur la question bosniaque occupe une place privilégiée dans les médias. Il est plus présent dans la presse islamique, tant celle de l'opposition que celle dite gouvernementale, où il constitue une des principales rubriques, que dans les autres organes de presse. Il n'en reste pas moins que du point de vue du statut, l'on a affaire à un dossier de "politique étrangère » même si le traitement diffère. La couverture médiatique comprend autant le suivi des événements - batailles, négociations - que les articles de fond relatifs aux développements de la question bosniaque; ou encore, les 
reportages en direct de correspondants qui, usant de l'argument d'autorité, feront valoir « la réalité bosniaque » telle qu'ils l'ont vue de leurs propres yeux.

Deux implicites non questionnés organisent l'ensemble de ce débat et expliquent largement le consensus autour de la question bosniaque. Le premier tient pour évident que la cause des Bosniaques est «juste» et que dans ce conflit il y a bien un agresseur et un agressé. Le second serait que cette cause «nous » concerne. Le "nous » renvoie ici à cette partie de l'élite intellectuelle et politique, directement impliquée dans la fabrication de l'opinion. En effet, même si la question bosniaque est traitée sous la rubrique « étranger ", elle en déborde en cela qu'elle n'est pas réductible à l'intérêt que l'on peut prêter à d'autres causes tout aussi justes. En dépit de ce consensus autour de la question bosniaque, plusieurs types d'oppositions plus ou moins marquées peuvent se dégager de la lecture de ce débat.

\section{Universalité/islamité ; le discours qui convient}

14 La première opposition est celle qu'on pourrait identifier dans la dichotomie universalité/islamité. Pour les uns, des diplomates notamment, la question bosniaque est à concevoir en termes de respect du droit international et des droits de l'homme. Cette mise en forme du problème est, semble-t-il, conçue à l'adresse des pays occidentaux, partagés sur l'attitude à adopter vis-à-vis de la question bosniaque : la présentation de celle-ci en termes islamiques ne pourrait que renforcer leurs suspicions et leur négativisme (al-Wafd, 18/12/1994). Dans cette même logique d'efficace du discours, s'inscrit la multiplication d'indices, de témoignages et de prises de position, visant à confirmer le caractère non religieux d'un conflit qu'il n'y a pas lieu d'énoncer en termes de solidarité islamique envers un peuple «musulman » et agressé en tant que tel. Il en est ainsi de la présentation du profil du vice-commandant de l'armée bosniaque: un serbe orthodoxe, dont la fille est mariée à un musulman bosniaque (al-Ahrâm, 20/12/1994). On soulignera également le fait que la question bosniaque mobilise en Occident certains secteurs de l'opinion au-delà des identités religieuses : par exemple, les «intellectuels juifs d'Europe qui ont été les premiers à soutenir les Bosniaques et à condamner le massacre, car eux savent ce qu'est la purification ethnique » (al-Wafd, 18/12/1994).

15 À l'inverse, pour d'autres, tant dans l'opinion islamique que de manière plus générale, il s'agit bien d'une guerre anti-islamique qui aurait pris, pour ainsi dire, le caractère d'une purification ethnique (al-Wafd, 17/12/1994). De la guerre anti-islamique au complot occidental, le pas est vite franchi : toutefois ce raccourci n'est pas sans poser de problèmes, y compris pour ceux qui s'en tiennent à la lecture religieuse du conflit. Fahmî Huwaydî - un journaliste proche de la mouvance islamique qui s'exprime régulièrement dans le très officieux al-Ahrâm - est le premier à souligner que la réaction des « sociétés civiles » occidentales en faveur des Bosniaques, contraste par sa vigueur avec celles timorées des États arabes et musulmans, plus soucieux de maintenir certains équilibres que de condamner leurs partenaires occidentaux (al-Ahrâm, 30/10/1995).

16 Dans les faits, si l'on examine de plus près cette opposition entre islamité et universalité de la question bosniaque, on s'aperçoit qu'elle semble se limiter au registre discursif. Opératoire, elle porte moins sur ce que l'on pense de la question bosniaque que sur ce que l'on doit en dire et les moyens de l'énoncer. On pourra ainsi paraphraser 
les tenants de la lecture « universaliste » en ces termes : « il ne fait pas de doute que ce soit une guerre contre des musulmans mais l'efficace du discours et sa "correction politique » interdisent de le dire.»

Par ailleurs, un autre type d'opposition se dégage qui porte sur les objectifs de l'intervention éventuelle: les différentes hiérarchisations renvoient à la question soulevée plus haut. Venir en aide à la Bosnie par les seules bonnes paroles n'est pas suffisant, tout le monde en convient. Cependant, pour les uns, à la survie physique des Bosniaques, c'est-à-dire à l'arrêt des combats, doit s'ajouter le «sauvetage de leur identité ", c'est-à-dire de leur islamité, dans la mesure où c'est bien cette dernière qui constituerait l'enjeu du conflit.

De cette manière de lire le débat égyptien sur la Bosnie il ressort deux formes de polarisation idéal-typiques. En forçant à peine l'analyse, il y a largement de quoi en faire deux blocs opposant universalité et islamité. Si l'on estime toutefois que l'intérêt heuristique de l'idéal-type - en tant que rationalisation provisoire - réside moins dans la conformité de ses vertus classificatoires avec un réel présumé, que dans la mise en évidence de ce qui précisément échappe à la typologie, l'on choisira de percevoir l'universalité et l'islamité comme deux constellations fluides. Pour les tenants de la lecture islamique, l'Occident "croisé » ne l'est pas toujours. Inversement, face à certaines situations, ceux pour qui le conflit bosniaque peut s'exprimer en termes universaux, c'est-à-dire non religieux, n'hésitent pas à dénoncer les affinités proserbes d'une Russie orthodoxe.

D'un côté comme de l'autre, l'on a pu dénoncer le double standard en matière de droit international, en évoquant, face à l'impunité des Serbes (et d'Israël), les sanctions subies par la Libye et l'Irak. Est-ce le standard que l'on dénonce ou sa duplicité ? Recourir à la dénonciation c'est aussi conforter le standard en le dissociant du mauvais usage qui en est fait. C'est que, "pour asseoir leurs mesures, [les protagonistes] prennent appui sur une équivalence générale traitée comme universelle » (Boltanski, 1990, p. 138).

20 Une même grille de lecture peut enfin produire des sens différents et l'humanisme apparent des universalistes instaurer de nouveaux particularismes.

$21 \mathrm{Au}$ nombre des représentations "exceptionnelles" de la question bosniaque, on retiendra ici celle d'un laïciste, le philosophe Fu'âd Zakariyya. Pour lui, nul doute sur l'identité de l'agresseur (serbe) et de l'agressé (bosniaque); pourtant, c'est l'ensemble du conflit qui marque la régression vers cet état de primitivité pré-moderne, celui des guerres religieuses, dans lesquelles seule la différence religieuse justifie l'acte de tuer. Façon pour notre auteur de renvoyer dos à dos ce qu'il interprète comme les volontés de puissance et les connivences objectives de l'extrémisme religieux, tant celui des États-Unis que celui des islamistes. Façon également de dénier aux agressés mais surtout à leurs alliés - ici, les islamistes égyptiens - le droit d'énoncer en termes religieux la nature d'un conflit que lui-même stigmatise comme étant... religieux.

Dans les faits, si l'on entreprend un examen systématique des emprunts réciproques entre les lectures universaliste et islamique de la question bosniaque, c'est à plusieurs Occidents que l'on a affaire ; ce qui fait leur multiplicité est moins leurs essences que les " échelles " mises en œuvre pour à chaque fois y désigner l'adversaire, voire l'ennemi (selon la distinction d'Alain Touraine) mais aussi, explicitement ou en creux, l'allié virtuel. 

généralement les qualificatifs " croisé », « impérialiste » et qui renvoie exclusivement à la stigmatisation; c'est, de toute évidence, la plus visible des constructions de l'Occident et cette visibilité conduit souvent à en éclipser d'autres.

Ainsi, identifier des États au sein de l'Occident c'est déjà introduire des nuances et qualifier dans les termes «croisé » ou «impérialiste » les États-Unis, c'est peut-être déqualifier l'Europe. Plus loin, faire ressortir les différences de position entre pays européens, en l'occurrence la France et la Grande-Bretagne, c'est également se situer à un niveau de totalisation inférieur. C'est admettre que les divergences entre ces deux pays à propos de la Bosnie relèvent d'un certain niveau de vérité, que celui-ci renvoie à leurs intérêts bien compris, à leur «style politico-diplomatique » ou à leurs traditions nationales respectives.

Considérons à présent les multiples manières de percevoir la position d'un État européen à l'égard de la question bosniaque. Il faudrait auparavant s'entendre sur ce qu'on place sous le terme de position: des « discours ", a contrario des "pratiques », que la seule situation (et l'analyse qu'ils en font) dicte aux commentateurs de retenir et de mettre en évidence. Il en est ainsi des déclarations respectives des présidents français Jacques Chirac et avant lui François Mitterrand. Déclarations que l'on a pu qualifier de prises de positions extrêmement positives; mais ces mêmes déclarations se prêtent tout aussi bien à une lecture inverse qui, cherchant à jauger une politique à l'aune de ses effets, n'y voit que des "discours » illustrant le "pseudo-engagement européen » en faveur de la Bosnie, c'est-à-dire, un phénomène purement médiatique, voire une manifestation ultime de "l'hypocrisie occidentale ", sinon française.

À un niveau moindre, c'est entre les États et leurs systèmes politiques que peut passer la ligne de partage. "Vive le peuple américain libre », peut-on lire dans l'éditorial d'un journal islamique. La veille, le Congrès américain venait de voter contre le président des États-Unis en faveur de la levée de l'embargo sur les armes et le journal al-Ahrâr met en évidence le respect que l'administration américaine doit au vote des congressistes ; saluer le vote des députés américains contre leur président c'était aussi - et le clin d'oeil est ici strictement interne - faire ressortir en creux ce que n'est pas le système politique égyptien. Enjeu interne également, la lecture qui opère une distinction entre les États et les sociétés civiles; en Europe, elles ont pu se mobiliser librement contre leurs États et pour la Bosnie, ce qui contraste avec le muselage en Égypte même, de la mobilisation islamique en faveur de la Bosnie.

Prenant la forme d'aide humanitaire, la solidarité des mouvements associatifs européens peut faire l'objet d'une dénonciation. L'aide aux petits orphelins bosniaques envoyés en Europe n'est pour certains, qu'une désislamisation déguisée, la poursuite par d'autres moyens de la purification ethnique. Ainsi, un même signe se voit attribuer simultanément et consécutivement plusieurs significations. Que l'on songe par exemple à la démission du délégué des Nations Unies aux droits de l'homme: éveil de la conscience occidentale ou preuve de plus contre les atrocités commises avec la complicité de l'Occident.

28 Rajoutons enfin que dans la construction de cet Occident, la ligne de partage passe quelquefois entre l'individu et ses appartenances présumées. Alors qu'en Europe même, certains engagements « cathodiques » pouvaient susciter des réactions allant de l'ironie à l'exaspération, en Égypte, et plus particulièrement dans la presse islamique, c'est encore « l'être-au-monde » d'un Bernard-Henri Lévy, intellectuel français juif et pro- 
bosniaque, qu'on prend le plus au sérieux, lorsqu'il s'agit de prouver que "même en Occident ", la Bosnie ne peut laisser indifférent.

On le voit, universalité et islamité sont deux registres ouverts aux emprunts, et dans lesquels les protagonistes du débat n'hésitent pas à puiser pour consolider des positions qui peuvent diverger. Cependant, dans ce que l'on a examiné ici, tout est affaire de situations; ce sont elles qui dictent le « discours qui convient $»^{1}$. Ce qui se passe dans les «fors intérieurs » est une autre question.

\section{Mesures et analogies ; l'espace-temps bosniaque}

Considérons à présent le débat à partir d'un autre point de vue. Présente dans les esprits, la Bosnie l'est certes, à plus d'un titre et sous de multiples formes simultanément, comme on a tenté de le montrer plus haut. Le problème serait plutôt de la situer sur la carte et c'est là que cette présence se signale par une absence. Où se trouve la Bosnie ? Force est de constater qu'en Égypte, la familiarisation avec ce coin de la planète est toute récente. Cela n'empêche pas d'en parler, bien au contraire. On ne parle pas pour autant - ou si peu - de ce que l'on est censé connaître ; l'ex-Yougoslavie - grand allié de l'Égypte dans les années glorieuses du non-alignement - qu'on aurait pu imaginer comme point de repère du débat à propos de la Bosnie, se présente comme une parenthèse vide. Silencieux également, le discours anticommuniste qui fleurissait au cœur du jihâd afghan, stigmatisait les athées comme premiers ennemis de l'islam, mais qui, semble-t-il, a perdu sa raison d'être, dès lors que son envers disparaissait avec la fin de la guerre froide.

Pour autant que l'on puisse se permettre de questionner une absence, cette " amnésie » donne à réfléchir. Sans aller jusqu'à lui attribuer une «fonction", on ne peut ignorer que le contournement de l'histoire immédiate de la Bosnie permet de faire l'impasse sur les explications causales du conflit qui s'y déroule. Est-ce parce que l'évocation de l'ex-Yougoslavie implique de trouver des explications raisonnables et autres que causales au déchaînement d'une violence irraisonnée? Est-ce encore parce que l'enchaînement des événements qui suit la disparition de l'État yougoslave ne devrait que fort peu à la formule fédérale qu'il a représentée et au statut des musulmans reconnus comme «nation »- au sein de cette formule ? Faut-il penser que l'évocation de l'ex-Yougoslavie est peu fonctionnelle dans renonciation de la solidarité avec la Bosnie ? En tout cas, cette occultation en dit long sur le travail de construction d'une auto-histoire nationale bosniaque (travail qui, à distance, implique des parties parfaitement extérieures à la " communauté imaginaire »), de production d'une lecture cohérente et légitime, et, à cet égard, les données attribuées à l'histoire longue semblent plus confortables que celles de l'histoire immédiate.

D'où la part signifiante que tiennent dans le débat, les renvois à et les analogies avec des situations précédentes, « déjà vues ", et dont la fonction est de déchiffrer le sens livré a priori - d'une histoire et de démontrer que la Bosnie n'est à la fois ni inédite ni anodine. Faisant partie de l'ex-bloc communiste, la Bosnie fut aussi une partie de l'Empire ottoman. Certaines lectures islamiques se réfèrent directement au démantèlement de l'Empire : l'Occident n'a pas fini de se disputer les dépouilles de l'Homme malade. La parenthèse titiste (en d'autres termes, l'État yougoslave), est réduite à une illustration d'un martyrologe commencé bien avant et se poursuivant ultérieurement. Dès lors que les ennemis des musulmans bosniaques sont identifiés 
comme serbes orthodoxes ou croates catholiques, il importe peu que la "désislamisation » soit le fait de la christianisation ou du communisme. S'il fustige « l'Occident croisé », le discours islamique n'en porte pas moins sur la grandeur passée de la Umma, sa misère actuelle et ceux qui en sont les responsables, voire coupables : la durée bosniaque est celle de la décadence et ce discours vise à la fois les sociétés et les gouvernants, notamment ceux des riches pays pétroliers du Golfe, autant coupables pour leur opulence que pour leur bonne conscience. Plus largement, ce sont les alliances occidentales des élites au pouvoir que l'on fustige.

L'on comprend mieux dans ce cas le rapprochement que fait un journaliste entre Sarajevo dévastée et Port-Saïd - ville égyptienne de la zone du canal détruite par les tirs israéliens -, Beyrouth ou Jérusalem, et les évocations en filigrane de l'Andalousie. Tout mène à croire cependant que les digressions sur la chute d'Al-Andalus (dite Reconquista pour d'autres), la Jérusalem occupée, Beyrouth déchiré ne font sens que par rapport à ceux qui les évoquent en Egypte sans avoir cette puissance évocatrice ailleurs. Au regard de ces analogies, le destin bosniaque n'est qu'une illustration de plus de l'état de la Umma et s'installe ainsi dans une durée de la persécution. Ici c'est un outsider $d u$ nom de Samuel Huntington qui fournit la grille de lecture de la Bosnie! Constructions d'analogies et/ou réactivation des imaginaires, 1e fait est qu'en Europe, et de manière synchrone, d'aucuns ont aussi fait appel à ces mêmes images inversées l'islam aux portes de Vienne - ou aux pesanteurs de l'histoire longue et ses séquelles ; une islamisation forcée au $\mathrm{XV}^{\mathrm{e}}$ siècle permettrait de "comprendre " la violence de l'agression que subissent les Bosniaques.

Situer le conflit bosniaque dans une durée autre est-il envisageable ? Non, si l'on s'en tient à l'abstraction que représente le stéréotype. Oui, si l'on prête attention à des représentations rares et qui par leur fonctionnalité restreinte dans les débats, n'auront pas l'occasion de se prêter (ou auront le privilège d'échapper) à la typification.

Dans cette perspective, la Bosnie est inscrite dans une durée qui n'est pas celle de l'antagonisme, mais plutôt celle des fractures. C'est ainsi qu'une reconstitution historique peut faire appel à la figure de Muhammad Abu 'Abdallah, dernier des Maures : témoin de la colère vengeresse des reconquérant, il témoigne aujourd'hui de celle des Serbes; c'est par ce biais également qu'elle peut évoquer la mobilisation exceptionnelle qu'a suscité la guerre d'Espagne, " un des moments les plus brillants de cette civilisation qui faisait croire que la tolérance était encore possible », aux dires de l'écrivain égyptien Muhammad 'Isa al-Sharqâwî. Ceux que l'Occident sacrifie par son silence complice sont les fidèles héritiers de Voltaire, Rousseau et Tolstoï ; et c'est sa vocation même qu'il trahit en laissant les nouveaux nazis de Belgrade s'emparer de Byron, Russel et Sartre, pour satisfaire aux injonctions d'un nouvel ordre mondial (série d'articles parus dans al-Ahrâm, notamment 04/08/1995 et 20/05/1995).

C'est encore le parallèle Madrid 1936/Sarajevo 1996 et le détour par Grenade que reprend l'écrivain espagnol Juan Goytisolo; pour ce dernier, ce qui explique la différence entre l'ardeur des brigades internationales de 1936 - 35000 combattants de toutes les nationalités dont Joseph Broz Tito - et la veulerie de 1996 - un pont aérien d'aide humanitaire - c'est, dans une certaine mesure, les préjugés anti-musulmans ; mais plus fondamentalement encore, c'est le fait que «la soumission du pouvoir politique à l'irrationalité des marchés a vidé la démocratie de sa substance - et [que] très peu de gens sont désormais disposés à mourir pour ce type de démocratie ». Le drame bosniaque résiderait dans sa mesure de retard par rapport à cette nouvelle 
réalité puisque «le gouvernement de Sarajevo combat au nom des mêmes principes civiques et politiques qui fondent les grandes démocraties européennes " (Le Monde diplomatique, février 1996).

Histoire commune ou historicité européenne ? La question reste posée mais quelle que soit la réponse, il semble évident qu'à ce point de l'analyse, il était peu pertinent d'écarter les convergences - qui sont autre chose que des effets de miroir -pour réifier les stéréotypes. Évident également, le fait que, pour significatives qu'elles soient, ces représentations rares pèsent peu dans des discours qui portent bien sur la Bosnie, mais dont les portées sont plus étendues et les fonctions plus larges.

\section{De la Umma à la nation : la rencontre avec l'altérité}

L'appartenance à la Umma n'abolit pas ce que l'on perçoit comme autre ; simplement, elle met en valeur des dimensions particulières. Celles qui mettent en jeu - en scène les appartenances communes et qui vont opérer comme marqueurs principaux quoique non exclusifs - de la différence, alors qu'elles devraient désigner la mêmeté. On voudrait explorer ici une autre dimension du débat autour de la Bosnie. La gestion de l'altérité - islamique ou non - renvoie à deux procédés qui là aussi sont inséparables et n'opèrent que de concert dans la tension; prétendre à une connaissance de l'autre, c'est tantôt ramener l'inconnu au familier, tantôt lui accorder (dans le doute) le bénéfice du relativisme culturel. Et dans ces deux modalités irréductibles, s'engage un retour sur soi.

Pour ce qui nous intéresse ici, et pour l'ensemble des protagonistes du débat sur la Bosnie, l'axe organisateur de la question « qui sont les Bosniaques?» est leur islamité. Question à laquelle on ne peut formuler de réponses qu'en mobilisant une norme ; prescriptive ou pseudo-descriptive, cette norme définit en creux les critères de l'islamité. Mais elle est elle-même marquée par des conditions d'énonciation, autrement dit, par l'efficace politique du discours de solidarité avec les Bosniaques. D'où la multiplicité des lectures de l'islamité bosniaque et des "niveaux de vérité " recherchés et mis en oeuvre. D'où également les glissements de sens entre islamité et "islamité politique ", si l'on peut dire ainsi, glissements assumés et niés en même temps.

Ils sont à la fois Européens et musulmans - autrement dit, musulmans à leur manière dit en substance une première lecture qui tend à s'inscrire en dehors du débat (alors qu'elle en est un des pôles); quelle que soit la réponse formulée sur la question de l'islamité des Bosniaques, cette question est elle-même doublement à rejeter parce que fausse d'une part - on ne dispose pas de « critère de mesure » de cet islam-là - et parce que politiquement inconvenante de l'autre; y répondre, c'est faire le jeu de ceux qui agitent l'épouvantail fondamentaliste pour justifier le désengagement moral de la communauté internationale; ou, a contrario, dénigrer la catégorie et la vider de son efficacité.

41 Dans une autre formulation, que l'on retrouve par exemple sous la plume de l'envoyé spécial du journal al-Ahâlî, organe du Rassemblement progressiste de gauche, le fondamentalisme ne peut avoir prise sur les Bosniaques parce qu'ils sont - puisqu'ils sont - Européens; le contact quotidien avec ces musulmans montre que leur islam est une expression nationale et non religieuse, réactive et non active. Cependant l'argumentaire déployé pour étayer cette opinion porte sur les signes de la «non- 
islamité » de la société bosniaque réelle, mesurée par des questions de forme et de conformité ; dire que ses modes alimentaires et vestimentaires sont européens est un euphémisme pour signifier le non-respect des obligations et des interdits religieux. Signalons au passage qu'au niveau interne, une telle lecture a pour effet de nier la relation privilégiée que les islamistes égyptiens prétendent instaurer avec la Bosnie et la mobilisation qu'ils organisent autour de cette question.

On n'est pas loin de ce que dit à ce propos un officiel bosniaque au Caire; en tant qu'Européens, les Bosniaques ne peuvent qu'être proches des lectures les plus tolérantes; de surcroit, leur islam " libéral » n'a pas pu être contaminé par le contact avec les combattants islamiques présents sur le terrain en raison des barrières linguistiques. Et l'on songe ici à ce reportage qui décrit la ville de Tusla comme terrain de luttes symboliques entre nouveaux fast-foods américains et styles de vie puritains des Mujâhidîn. En somme, l'islamité des Bosniaques comme supplément d'âme ou comme nationalité, ces deux termes renvoient à des perceptions voisines.

Ce ne sont pourtant pas les seules et pour ceux qui pensent la Bosnie avant tout à l'intérieur des frontières symboliques de la Umma, la question se pose en d'autres termes. Il s'agit de termes régionaux et c'est l'ensemble des musulmans de cette partie du monde - délimitée en termes d'ex-bloc communiste - qui est concerné, Bosniaques compris. Cependant, il ne s'agit plus seulement de munir ces musulmans de corans, d'imams, ou de bourses d'études religieuses : la Umma se mettant à l'heure du village mondial, le message de l'islam émis à partir du centre musulman vers ses "périphéries", passera d'autant mieux qu'existeront des satellites, des antennes paraboliques et des chaînes de télévision que des promoteurs financiers et des spécialistes de la communication "islamique " prévoient de mettre en place (Majallat al-Azhar, mars 1995). Ces "réseaux» d'un nouveau genre ne sont pas une simple sophistication technologique des liens ancestraux entre centre(s) et périphéries de la Umma. Assez paradoxalement, c'est en faisant face à l'occidentalisation sur ce qui est censé être son terrain de prédilection - la "cocalisation » du monde, selon le même Fahmî Huwaydî, qui dénonce par ce néologisme l'hégémonie d'un style de vie américain (al-Ahrâm, 10/10/1995) - que la réislamisation des périphéries s'inscrirait au cœur de la «mondialisation ».

Une fois cet "espace à reconquérir » délimité, resterait à fonder la spécificité d'une islamité bosniaque. C'est chose faite dans la relation de voyage en Bosnie et Croatie - on y reviendra - d'un cheikh d'al-Azhar. L'islam bosniaque aura résisté à toutes les tentatives d'acculturation: cet espace a constitué un lieu de confrontation privilégié avec les ennemis de l'islam, et ce, du fait que les Bosniaques ont été, de tous les musulmans d'Europe, les plus attachés à leur islam. S'ils sont ainsi, c'est parce que dès les origines, avant même leur islamisation, leur forme de chrétienté s'est distinguée des Églises orthodoxes et catholiques et les a poussés vers une conception de l'unicité qui expliquerait aujourd'hui leur attachement à l'islam (Qutb, $1413 \mathrm{H}$ ). Ces musulmans " aux yeux bleus et aux cheveux couleurs de paille ", se seraient donc distingués par la continuité de leur attachement à l'islam.

En parallèle pourtant, sont identifiés des « faits » qui sont autant de signes de « retour à l'islam »; port du voile, construction de mosquées, respect du jeûne, regain d'intérêt pour l'histoire, et le remplacement des imams des mosquées communistes par une génération plus jeune et sincère dans son islam, tels sont ces signes repérables qu'il convient de consolider en donnant à l'islam bosniaque un nouveau contenu. Au nombre 
de ces signes l'on ajoutera également la fin des mariages mixtes, le renforcement de la famille et la baisse de la criminalité. Dans les faits, l'inventaire des signes est autant celui, classique, des retours à l'islam que celui qui permet de décrire de manière générale les utopies d'après-guerre ; et l'image donnée est celle d'une société soudée et " purifiée " par la lutte, ignorant l'écart entre masses et élites, et qui, ayant payée au prix fort la sauvegarde de son "identité islamique», ne peut que s'y attacher, se reconstituant enfin sur la base d'une parfaite adéquation avec cette identité. C'est du moins une manière de voir les choses à partir de l'Égypte. Resterait à se demander si les intéressés partagent cette vision.

Examinons de ce point de vue ce qui ressort d'un colloque organisé en 1992 par l'université Ibn Sa'ûd. Côté saoudien, il s'agissait de soutenir les Bosniaques dans leur islam et de faire de cette région le coeur et la base de départ de l'islam en Europe. La Présidence des musulmans yougoslaves, elle, était certes porteuse d'une «demande d'islam » : des mosquées, des savoirs religieux, de la prédication mais aussi des fonds. Cependant, cette demande n'en était pas moins compatible avec une autre revendication bosniaque: "Nous sommes des Européens de souche qui avons volontairement embrassé l'islam dès le XV $\mathrm{XV}^{\mathrm{e}}$ siècle. » (Al-Semari, 1992)

Islam d'Europe versus islam européen, c'est là que les lectures en provenance du centre autoproclamé de la Umma semblent entrer en compétition avec celles des intéressés, c'est là que les frontières de la Umma se présentent non plus comme donnée mais comme construction, en interférence avec d'autres «cités». Pour le centre autoproclamé, « l'islam d'Europe » est une ruse de l'histoire et de la géographie et son inscription territoriale ne saurait en rien préjuger d'une identité spécifique des "musulmans d'Europe». Pour ces derniers, il semble en être autrement et «l'islam européen » s'inscrit volontairement dans une appartenance occidentale et relève d'un tout autre registre que l'identification islamique: les deux registres n'en sont pas moins compatibles. C'est dans ces termes qu'on peut, semble-t-il, interpréter une partie des débats sur la Bosnie et plus encore les non-dits et les silences de ce débat. Cette revendication de double inscription semble heurter «l'universel islamique » tel que perçu et exprimé par certains secteurs de l'opinion en Égypte.

De la même manière, demeurent incompris certains éléments jugés antinomiques avec ce que serait une identité bosniaque islamique : ainsi du vote bosniaque pour les partis de gauche ou plus tardivement, de la confiance que pouvaient encore accorder les Bosniaques «musulmans » aux Croates « catholiques». A posteriori, les conflits entre ces deux groupes, puis l'issue négociée, considérée comme favorable aux Croates d'aucuns feront le rapprochement entre Camp David et Dayton - seront utilisés comme autant de justifications, sur le mode du «on vous l'avait dit». Serbes et Croates, bonnets blancs et blancs bonnets.

En définitive, le problème soulevé par toutes ces oppositions semble renvoyer à l'islamité « réelle » des Bosniaques : s'agit-il de les islamiser ou de les réislamiser? L'on comprend que la question ne puisse pas se poser de manière explicite ${ }^{2}$ : porter un regard critique sur l'islamité des Bosniaques rendrait caduc le soutien exprimé en termes d'appartenance à une même Umma, d'autant qu'il est d'usage d'affirmer que celle-ci s'enrichit de la diversité de ses composantes.

50 Parti pour assurer les Bosniaques du soutien d'al-Azhar, centre du monde musulman, le périple de cheikh Jamâl Qutb est une quête des signes de l'islamité bosniaque. Sa relation de voyage illustre de bout en bout l'exercice auquel il a été fait allusion plus 
haut ; déchiffrer et jauger l'inconnue - l'islam des Bosniaques - à l'aune du familier que l'on connaît ou que l'on croit connaître - un procédé qui est loin d'être spécifique à la Umma, et qui, simultanément, autorise le retour sur soi.

51 Le «soi » est en l'occurrence l'émissaire officiel d'al-Azhar qui cumule par ailleurs plusieurs attributs : le dignitaire religieux est député au Parlement. Au moment où il relate son témoignage bosniaque, il est l'une des principales figures de l'Establishment religieux comme de l'organisation des Frères musulmans. Depuis 1995, cette dernière appartenance lui a valu une peine de prison. Sa relation de voyage enfin, est publiée dans la série d'ouvrages diffusés en accompagnement du mensuel al-Azhar, organe officiel de l'institution du même nom. Autant d'indices qui ne sont pas anodins si l'on veut situer le lieu à partir duquel on peut s'exprimer sur la Bosnie en Égypte. Ce lieu ne recoupe pas le découpage simpliste entre parole officielle autorisée et parole contestataire interdite, mais il se présenterait comme agencement astucieux de scènes et de discours.

L'on pourrait ainsi renvoyer à une division fonctionnelle des tâches, les deux discours qui rendent compte de la présence égyptienne en Bosnie. À la presse, toutes tendances confondues, la couverture des hauts faits du contingent égyptien de l'Onu présent sur le terrain. Ce dernier constitue - du moins à travers ce qu'en disent diplomates et correspondants de presse - un objet de fierté nationale et la réplique exacte de ce que l'Égypte a "vocation" d'être: un pays musulman, une société moderne, un État respectueux des engagements internationaux. Le professionnalisme que requiert ses tâches techniques - maintenir en état l'infrastructure de Sarajevo - la neutralité imposée par son statut onusien et, enfin, le fait qu'il y ait bien un agresseur et un agressé - les bosniaques l'étant au titre de musulmans - fait du contingent une sorte de microcosme de cette « exemplarité égyptienne ». Sur le terrain, le contingent réussit en quelque sorte à concilier autour de lui l'inconciliable : « confiance de l'Onu, amitié des Bosniaques et respect des Serbes». L'exemplarité nationale semble tenir dans cet équilibre délicat entre la solidarité et la neutralité qui, l'une comme l'autre, vont de soi. Le contingent s'affirme comme musulman: des repas pour les Bosniaques pendant le Ramadan ou encore le respect du jeûne sunnite selon la tradition prophétique (lundi et jeudi) par ses membres et la distribution du surplus de vivres à la population bosniaque. Sans enfreindre la neutralité, le recrutement de personnel local par le contingent privilégie les Bosniaques, alors que les autres forces de l'Onu privilégient les Serbes ou les Croates. Sont mis en valeur les aspects d'une sociabilité spécifique: les Égyptiens sont le seul contingent apprécié par les Bosniaques dont les sentiments à l'égard des forces de l'Onu ne sont pas particulièrement chaleureux.

Cette exemplarité nationale "officielle» ne semble en rien différer de celle « officieuse » dont rend compte le cheikh Jamâl Qutb, et que représente le Comité de secours islamique, fruit des mobilisations associatives islamiques en Égypte et dans le monde musulman. Les organisations de secours islamiques présentes en Bosnie et dont des branches sont implantées dans plusieurs pays, semblent porter toute l'ambiguïté constitutive des ONG internationales et de l'ordre mondial dans lequel elles s'inscrivent, à mi-chemin qu'elles sont entre organisations étatiques et paraétatiques ; le Comité "égyptien» de secours à la Bosnie a été fondé (tout comme pour l'Afghanistan) à l'initiative des Frères musulmans, au sein du syndicat des médecins. Et la relation du voyage quasi officiel de l'émissaire "pour témoigner de la présence d'alAzhar, espoir des musulmans » est une présentation des activités du Comité de secours 
islamique - constitué, faut-il le rappeler, de membres potentiels d'une frange non légalisée de l'opposition.

Deux représentations identiques de l'exemplarité nationale et islamique, à cette exception près que la seconde, tout en assurant, comme son homologue officiel, aide médicale, aide alimentaire et soutien psychologique "islamique", renforce le volet «moral»: distribution de voiles à celles qui les ont «perdus » pendant les combats, organisation de l'accueil des orphelins afin de ne pas laisser cette mission aux ONG occidentales, qui cherchent ainsi à détourner les enfants de leur islam.

Cette incursion dans les deux formes de la présence égyptienne en Bosnie était nécessaire pour mieux situer le regard que porte le cheikh Jamâl Qutb sur la Bosnie : celui qu'un centre autoproclamé de la Umma porte sur sa périphérie. L'on comprend qu'au Caire même, sa première démarche fut de préparer le voyage en rencontrant un ami dont l'épouse est "slave»(du Montenegro!), afin de "connaître les us et coutumes» de ce pays. L'on comprend également qu'une fois sur place - «Croatie, terre ottomane et sœur de l'Andalousie » - le premier signe repéré par notre émissaire et qui le réconforte fut la présence de mosquées. L'on comprend enfin ce qu'après coup il dit ne pas comprendre : que la restauration d'une mosquée démolie par les projectiles à Zaghreb puisse être encadrée par la municipalité croate; que ce soit la télévision croate qui retransmette la prière de la fête du sacrifice et que dirige l'émissaire d'alAzhar auprès des Bosniaques.

Quêtant les signes de l'islamité, le cheihk Jamâl Qutb décrit l'habit d'une jeune femme médecin comme étant "celui des Mujâhidîn»: elle porte en effet une parka de combattant kaki (le type même du vêtement universel) agrémentée pourtant d'un brassard aux signes de l'islam (croissant vert et : » il n'y a de dieu que Dieu »).

Dans les faits, les signes repérés transparaissent moins dans les propos que dans les photos couleurs qui les accompagnent tout au long de l'ouvrage; fillettes voilées et souriantes, combattants affublés de bandanas, mosquée bondée, stocks de vivres en provenance d'Égypte, et sur toutes ses photos, l'émissaire égyptien lui-même. Autant de signes du " retour de l'islam dans une Bosnie qu'il n'a jamais quittée ». Par ailleurs, le message transmis aux Bosniaques, est celui d'une indéfectible solidarité : «C'est alAzhar même que les Serbes foulent de leurs pieds. »

\section{L'altérité chez soi ; Izetbegovic au pays d'Al-Azhar}

Reçu au Caire en janvier 1995 pour la remise du prix saoudien du roi Fayçal, celui que la presse islamique surnomme al-Mujâhid nepouvait- diplomatie oblige -que se féliciter des soutiens égyptiens et saoudiens accordés à la lutte de son peuple. Pour des considérations du même ordre, semble-t-il, l'allocution qu'il prononça lors de la remise du prix portait sur l'apport des Arabes à la civilisation islamique. Cependant, ce sont surtout ses dernières phrases que la presse islamique s'empressa de mettre en exergue :

«Nous n'avons pas le respect du temps, alors que la prière, fondement de notre culte, doit se faire à des moments précis [...]. Nos grandes villes sont sales alors que nous appartenons à un monde (musulman) dans lequel propreté et hygiène sont partie intégrante de la religion [...]. Comment se fait-il que la dimension spirituelle de la religion se transforme en croyances magiques alors même que le Coran est par définition un livre de réflexion? [...]. D'où nous vient enfin le culte des chefs dans une croyance qui témoigne qu'il n'y a de dieu que Dieu? [...]. Qui répondra à ces 
quatre questions aura bien mérité le prix Fayçal de l'année prochaine. » (al-Sha'b, 17/1/1995) eommunistes en raison de son attachement à l'islam. Soulignant le caractère pédagogique de sa da'wa (prédication) et de son action, certaines plumes dans la presse islamique vont jusqu'à en faire un disciple de Hasan al-Banna, fondateur des Frères musulmans. Menant la lutte de son peuple, Izetbegovic est à mi-chemin entre le combattant moderne et le calife des premiers temps. Cependant, pour que cette représentation porte tout son sens et son efficace, l'image que l'on donne de lui est surtout celle - inversée - des dirigeants des pays musulmans de nos jours. Le président bosniaque se caractérise par son abnégation. «Il partage la vie quotidienne de ses hommes, se rend à la mosquée à pied cinq fois par jour, accompagné simplement par un garde du corps, et n'utilise pas de voitures vertigineuses » (al-Nûr, 21/12/1995). Pour qui connaît la valse des cortèges officiels dans les rues du Caire, la remarque n'est pas anodine. Izetbegovic est introduit dans une historicité qui excède le cadre de la cause dont il est le porte-parole. Ainsi devient-il la figure emblématique du chef tel qu'il devrait être et le prétexte qui permet de pointer ce que ne sont pas les leaders du monde musulman.

61 Il n'en cesse pas pour autant d'être président de la Bosnie et c'est à travers les réceptions égyptiennes de ce qu'il dit à propos de son pays que l'on peut entrevoir les multiples «remises en ordre ", nécessaires pour concilier ses paroles avec ce que des Égyptiens voudraient continuer à croire à propos de la Bosnie en tant que partie de la Umma - le tout et la partie étant vus du Caire. Avec la visite d'Izetbegovic coïncide la parution en langue arabe de L'Islam entre Orient et Occident (Al-islâm bayn al-shark wa-lgharb, 1995) œuvre de prison d'Izetbegovic à propos de laquelle ses éditeurs organisent un débat. À ce dernier participent essentiellement des intellectuels proches des mouvances islamiques. Et ce débat est l'occasion d'un croisement des regards, qui porte autant sur l'ouvrage en question, que sur la situation bosniaque, reflétant la manière dont des intellectuels islamiques égyptiens ont pu lire la Bosnie à travers l'ouvrage ou, inversement, déchiffrer l'ouvrage à partir de la situation bosniaque. Double lecture qui, semble-t-il, se déroule dans les deux sens sur un mode heurté.

L'Islam entre Orient et Occident ne porte pourtant pas sur la Bosnie et il n'y est fait aucune allusion à ce pays. Tel que le présente son auteur, ce n'est pas non plus un traité de théologie, pas plus qu'il n'y s'agit de présenter l'islam de manière didactique. Il y est "simplement» question de l'islam comme "vision du monde», exprimé dans un langage accessible aux jeunes générations. La particularité de cet ouvrage et l'effet d'étrangeté qui accompagne sa réception tiennent moins à ses prémisses qu'à son 
argumentaire. Les premières sont relativement simples et consensuelles : l'islam est l'unité bipolaire du matériel et du spirituel et c'est dans ces termes qu'il se définit dans son rapport aux monothéismes; il n'est pas tant mesuré à l'essence du christianisme et du judaïsme, mais à ce qu'ont pu être les conditions sociales et historiques de construction a posteriori des images de Moïs et de Jésus par leurs adeptes; leur efficace historique tient moins à ce qu'ils furent qu'à ces images. Aussi Izetbegovic réserve-t-il le terme « religion » à l'expérience des sociétés occidentales : loin d'être une continuité des croisades et des guerres de religion, l'incompréhension à l'égard de l'islam dans ces sociétés s'inscrit, d'une part, dans leur expérience même de la laïcisation et, de l'autre, dans l'incapacité des langues européennes à rendre compte de la double dimension contenue dans les mots clés de l'islam. L'homme est-il condamné à faire le choix entre l'au-delà et l'ici-bas ? L'utopie terrestre peut-elle être habitée par des hommes et non des individus sans visage? et peut-elle prendre les traits du «royaume de Dieu sur terre »? L'islam qui n'est ni mouvement social ni spiritualité éthérée, ni « religion » ni "style de vie », ni enfin la moyenne arithmétique de ces extrêmes, prend acte de la dichotomie, la comprend et tend à la dépasser. Tout comme l'homme est l'union du corps et de l'esprit, l'islam est l'union de la religion et du système social. Pour l'individu - dont la compréhension dépassera toujours la somme de toutes les sciences réunies- et pour la société - dont le modèle idéal est la communauté médiane (Umma wasaf) l'islam est quête d'équilibre.

La première partie de l'ouvrage examine au travers de thèmes comme l'art, la tragédie, la morale ce que l'auteur identifie comme "la saisie chrétienne de la conscience humaine »; le socialisme relève également de cette même saisie, puisque l'alternative posée, celle de l'utopie terrestre, est la même; seul diffère le choix et le socialisme a chrétien n'est qu'un christianisme inversé nourri aux mêmes valeurs. Dans sa deuxième partie, l'ouvrage traite de l'islam comme alternative. Moins convenue et plus brève est la troisième partie de l'ouvrage qui a pour titre «La sortie de l'islam; la troisième voie ». Ce qu'Izetbegovic identifie comme la tradition anglo-saxonne réformiste (qui n'a pas connu de rupture violente entre l'avant et l'après du siècle des Lumières) constitue un islam «de l'état de nature » européen. Ce n'est pas tant Marx qui a troublé les classes moyennes britanniques (celles qui ont intégré le réformisme social à leurs programmes), que Charles Darwin. Adam Smith, J. S. Mill ou G. B. Shaw, pourraient être des penseurs musulmans, en cela que pour eux l'éthique s'inscrit au cœur même de la quête individuelle de l'intérêt et du plaisir. Islam égale démocratie sociale? C'est bien de cela qu'il s'agit. Tout en articulant cette triple contingence entre un message révélé, sa traduction dans une expérience historique et le patrimoine de l'humanité, Izetbegovic ne nous dit pas s'il destine cet islam-là aux non-musulmans ou à l'ensemble des hommes - considérations qui sortent de notre propos.

Ce qui nous intéresse en revanche, c'est l'examen des réceptions possibles de ce discours par un public d'intellectuels islamiques égyptiens. Familiers de Thomas Carlyle, il ne leur est pas difficile d'admettre un Monod, un Maritain ou un de Chardin, dans le palmarès des "savants croyants". Pas plus qu'ils n'auront de difficultés à intégrer dans leurs argumentaires la cérémonie du Potlatch comme preuve d'un sens inné du divin chez les primitifs, même si ce n'est pas exactement ce qu'a voulu en dire Marcel Mauss. Qu'Izetbegovic se réfère à Luckas ou Mao pour argumenter à propos de la pauvreté du marxisme après Lénine, qu'il pourfende Engels à propos de la famille de l'intérieur même de son système de pensée, cela n'est pas sans exercer un effet d'étrangeté sur l'auditoire égyptien, mais il s'agit après tout du bagage culturel d'un 
intellectuel de l'Est. Bagage qui, par ailleurs, est loin d'être inconnu à certains intellectuels islamiques lesquels, il n'y a guère longtemps, se nourrissaient aux mêmes sources. Qu'enfin ces intellectuels soient potentiellement ouverts aux compromis historiques les plus "contre-nature " et aux synthèses intellectuelles les plus inattendues, cela non plus n'est pas à exclure.

On notera ici que la revue al-Azhar n'y voit pas malice qui publie sur quatre numéros une présentation non problématique de l'ouvrage - l'aura de son auteur n'imposant pas d'autres formes de justification. Il en est autrement pour les intellectuels islamiques modernistes. S'il faut attribuer une part de leur incompréhension tant aux difficultés de la translation d'un système de références à l'autre qu'à celles de la traduction (la version arabe est traduite en Allemagne à partir d'une traduction anglaise de l'original serbo-croate), c'est ailleurs, dans la situation historique elle-même, que se situe plus fondamentalement cette incompréhension (à moins qu'il ne s'agisse, à l'inverse, d'un surcroît de compréhension) : L'Islam entre Orient et Occident est une œuvre de prison dont la rédaction est antérieure à l'éclatement fédéral, d'où l'impression d'anachronisme. Pour s'en justifier pleinement auprès de son auditoire (la renier?), Izetbegovic doit invoquer les prudences imposées par la censure et l'autocensure yougoslaves.

66 Pour les intellectuels islamiques qui ont lu l'ouvrage et le discutent, Izetbegovic se permet de philosopher dans un contexte où philosopher n'est pas de mise. Il semblerait pourtant que ce ne soit ni par rapport à ses idées en tant que telles, ni vis-à-vis de ses références en elles-mêmes qu'ils maintiennent la réserve, mais bien eu égard à leur mobilisation. De ce fait, L'Islam entre Orient et Occident ne constitue pas le « discours qui convient ", Izetbegovic se gardant d'y désigner explicitement l'ennemi. Certes, ses lecteurs n'ignorent pas les circonstances de rédaction de l'ouvrage. C'est pourtant la même thématique - désignation de l'ennemi - qui est mobilisée dès lors qu'il s'agit de la Bosnie et Izetbegovic - que d'aucuns ont pu rapprocher du calife Omar ou de Hasan al-Banna - s'en explique.

67 Côté égyptien, l'identité bosniaque est posée comme une donnée du temps long, sauvegardée par l'attachement à l'islam. Le nationalisme islamique à travers lequel elle s'exprimerait aujourd'hui serait en quelque sorte l'accomplissement d'un dessein historique qui n'aurait que trop tardé, mais qui en revanche réaliserait dans sa forme la plus parfaite, le recoupement entre appartenance nationale et appartenance religieuse. Et cela, pour peu que les Bosniaques veuillent bien prendre conscience que l'islam est plus qu'une nationalité. Ainsi et partant de ces «données » et de ces "potentialités ", l'on comprend mal l'attachement qu'ont pu afficher les leaders bosniaques à un État unifié. Dans les faits, ce que l'on comprend mal c'est plus précisément qu'au milieu de tant d'adversité, les Bosniaques refusent de couper les amarres avec l'Europe et plus généralement avec l'Occident. La Bosnie-symbole est perçue à la fois comme une « terre neuve " - c'est-à-dire une terre de missions - et d'utopie - et comme une donnée ancestrale. En dépit du nouvel ordre mondial ou à cause de celui-ci, au cœur de l'Europe, sur un territoire qui fit partie de l'Empire ottoman, la Bosnie, État musulman, réunirait toutes les composantes nécessaires qui feraient d'elle une chance pour la Umma. Encore faut-il que les intéressés en soient conscients. Et que leurs visées traduisent les exigences d'un modèle de l'identité islamique épurée.

Pour Izetbegovic, le seul donné qui tienne est bien la superposition des cartes. «Quand les nationalismes émergent au XIX ${ }^{\mathrm{e}}$ siècle, ils s'arc-boutent sur les religions existantes, 
les orthodoxes devenant serbes et les catholiques croates. Jusqu'il y à quelques années, les musulmans n'avaient pas de définition nationale ; nous nous sommes auto-désignés comme Bosniaques, l'islam est notre religion et la Bosnie notre nationalité, mais la Bosnie est un mélange » (al-Sha'b, 17/1/1995). Au même titre que celle affichée par les autres groupes, l'identité des Bosniaques serait ainsi une construction. Izetbegovic présente cette identité comme un travail d'élaboration dicté par une conjoncture spécifique : s'affirmer comme Bosniaque musulman n'était qu'une réponse obligée aux mutations nationalitaires des autres groupes, voire un pis-aller. Cependant, tous les Bosniaques ne sont pas musulmans et l'identité bosniaque transcende les clivages ethniques. Pour Izetbegovic, la superposition des cartes et des appartenances ethnicoreligieuses que l'on pourrait percevoir comme une contrainte est un ressort et c'est bien dans ce sens - celui de la pluralité - que la Bosnie serait, de son point de vue, la chance de la Umma. Izetbegovic énonce - au cœur du pays d'al-Azhar - ce que serait le rôle d'exemplarité de ce qu'il identifie comme "l'islam européen " $^{3}$ ni base d'islamisation ou de ré-islamisation, la Bosnie européenne et musulmane se donnerait pour mission de «témoigner du vrai visage de l'islam et de sa tolérance ». La Bosnie, Umma wasat? L'implicite est-il que dans d'autres régions du monde musulman, c'est une fausse image de l'islam qui est donnée ? Dans une Égypte qui se pense en tant que cœur du monde musulman, les propos d'Izetbegovic étaient-ils recevables? Vue de Bosnie, l'Égypte se retrouverait-elle à la périphérie de la Umma? Et de là, à la périphérie du monde?

Ces exemplarités en compétition semblent renvoyer, au premier chef, aux ancrages «nationaux » respectifs de nos interlocuteurs. Pour Izetbegovic s'exprimant au Caire, l'exemplarité de la Bosnie est d'abord signifiante par rapport à son environnement européen et c'est à ce titre qu'elle retrouve sa place dans un monde musulman. Pour ses interlocuteurs, cette exemplarité tient dans la résistance des Bosniaques à un environnement destructeur, voire, dans la déconnexion d'avec celui-ci. Il reste que si Jérusalem occupée et Beyrouth déchiré s'inscrivent dans un espace signifiant, celui-ci n'épouse pas forcément les contours de la Umma. Qu'à l'intérieur même de celle-ci, et à moins de se retrouver dans les tranchées (et encore), arabité et «bosnitude » sont autant de "cités ». Qu'enfin, vue du centre auto-proclamé de la Umma et située ainsi à sa frontière - du moins à l'une de celles qu'elle partage avec l'Occident - la Bosnie mobilise, émeut et dérange. Que les Bosniaques eux, sont pour le moment aux prises avec d'autres problèmes.

\section{Solidarités ; l'agir de la Umma}

De ce qui précède, on peut relever qu'en définitive, les dynamiques à l'oeuvre dans la perception de cet autre même qu'est la Bosnie vue du Caire, ne sont pas d'une singularité radicale; on est toujours à la périphérie d'un centre. C'est dans les axes organisateurs de cette perception qu'on peut relever ce qui serait le propre de la Umma - l'islamité - et plus encore dans l'intensité de l'investissement tactique et émotif dans le sens Caire/Sarajevo. Faire la part des choses entre ces deux facettes de l'investissement est en revanche impossible ; l'on ne peut pas en déduire que la Umma n'est pas une catégorie efficace, pas plus qu'on ne peut prétendre que ceux qui s'y reconnaissent soient agis par cette catégorie. L'on peut tout au plus relever le poids des 
conjonctures qui contribuent à donner leurs formes à ces modalités indivisibles de l'investissement pour une « cause ».

La profonde empathie que des voix émanant de «l'opinion publique » égyptienne ont exprimé envers la Bosnie, n'est pas exempte d'instrumentalisations: celles-ci conduisent à réactualiser les contours et les contenus de la Umma. Au début des années quatre-vingt, c'est aussi au nom de celle-ci que l'on mobilise. Pour l'Afghanistan, contre le communisme, la mobilisation reçoit le parfait assentiment du pouvoir égyptien dont les relations avec les mouvements islamiques sont encore à l'accommodement. Et c'est enfin en termes de jihad que s'exprime la campagne de mobilisation quasi officielle en Égypte. Un témoignage de Kaboul, publié en 1993, rapporte que les combattants volontaires auprès des Afghans ont quelquefois transposé leurs conflits internes sur le terrain, opérant par là même un classement parmi les groupes afghans les plus dignes de soutien logistique et financier. Qu'une compétition certaine opposait les radicaux plus proches des lignes de combat aux «modérés ", plus enclins à gérer les services et les lignes arrières. Que des "coutumes locales" afghanes leur sont apparues comme incompatibles avec l'islam: qu'en revanche certaines des idées que les Égyptiens auraient véhiculées - comme les karamât al-shuhadâ' (signes ou miracles accomplis grâce à l'intercession des martyrs) - ont été peu appréciées par les combattants afghans. Qu'aspirant au martyre, certains des volontaires se sont rendus coupables d'audaces guerrières non justifiées (Daraz, 1993). A posteriori, comme pour beaucoup de " grandes causes ", les luttes fratricides des princes de la guerre ont conduit plus d'un à réfléchir sur le sens de son engagement. Les leaders des Frères musulmans impliqués par la suite dans la réconciliation précaire entre les groupes afghans n'ont pas caché leur amertume face à ce qu'était devenue la «cause». Et au sein même de l'opinion islamique, les critiques ne manquent pas qui ont mis en regard la tiédeur de l'Arabie Saoudite en Bosnie et l'empressement naguère manifesté à l'égard d'une "cause » afghane que soutenaient les États-Unis.

72 En 1995, l'Union soviétique n'est plus. Les relations entre le pouvoir égyptien et les islamistes - y compris la composante participative - en sont au conflit ouvert. Le Comité du secours islamique, perçu et dénoncé comme l'un des bras de $\mathrm{P}$ » Internationale islamique » est accusé de financer le terrorisme. Enfin, même si l'on sait que des combattants égyptiens sont partis sur le terrain à Zaghreb ou Sarajevo - et l'on appréhende le retour des "vétérans bosniaques ${ }^{4}$ après celui des " vétérans afghans " - il reste que, tant du côté du pouvoir que de celui des opposants, la campagne de mobilisation aura emprunté à un tout autre registre. En phase avec l'ordre mondial, c'est en termes d'aide humanitaire qu'elle s'exprime. En 1996, "mourir pour l'islam » en Bosnie, ressemble peu à ce qu'était en 1980, « mourir pour l'islam » à Kaboul.

La Bosnie aura-t-elle une signification dans la socialisation d'une génération de militants islamiques, comme a pu l'être l'Afghanistan? Il est encore trop tôt pour le dire et pour longue qu'elle soit, la mémoire de la Umma n'en est pas moins sélective : oubli et distanciation sont synonymes. Dans les représentations égyptiennes de la question bosniaque, si la Umma peut expliquer le sentiment d'identification, elle n'occulte pas pour autant le travail de la raison et ces deux dimensions sont plus imbriquées qu'elles ne sont simplement juxtaposées. Et l'appel que d'aucuns qualifieraient d'«identitaire» de la Bosnie en 1996 n'est ni plus vrai ni moins irrésistible que ne l'était l'appel "citoyen» de l'Espagne en 1936. Ce qui fait la différence entre mourir pour l'islam et mourir pour la démocratie tient moins à la 
nomination du Dieu que l'on sert, c'est-à-dire aux utopies, ou aux dispositions sacrificielles des " croyants ", qu'il ne tient au regard de ceux qui observent.

Simplement, il est des situations où le déséquilibre des forces en présence est tel, que l'égalité des compétences entre les acteurs engagés dans un conflit (principe fort louable par ailleurs), a du mal à résister aux faits. Il est concevable que dans des situations où la domination est écrasante, « la raison » du plus fort soit potentiellement dominante. Au point de fournir à la résistance ses grilles de déchiffrement et de désignation de l'ennemi. Pour l'observateur, la difficulté serait de résister à la tentation de prendre pour du réel la «vision des vaincus ». C'est ce que l'on a tenté tout au long de ses pages.

\section{BIBLIOGRAPHIE}

ANDERSON Benedict, 1983, Imagined Communities, Reflections on the origins and Spread of Nationalism, London, Verso.

BADIE Bertrand, 1996, « Les politiques étrangères peuvent-elles encore être autonomes ? ", L'État du monde 1997, Paris, La Découverte.

BEN ACHOUR Yadh, 1991, « Le nationalisme arabe sans peur », Pouvoirs, n 57, p. 85-92.

BOLTANSKI Luc, 1990, L'amour et la justice comme compétences, trois essais de sociologie de l'action, Métailié.

DARAZ 'Isâm, 1993, Al-'A'idûn min Afghanistân, ma lahum wa ma 'alayhim (Les vétérans de retour d'Afghanistan ; le pour et le contre), Le Caire, 1993.

IZETBEGOVIC Alija, s.d., L'Islam entre Orient et Occident, [édition égypto-kowétienne aux éditions al-Shuruq pour Le Caire, dans la collection « Fenêtre sur l'Occident »].

MITRI Tarek, 1993, « La Bosnie-Herzégovine et la solidarité du monde arabe et islamique », Maghreb/Machrek, $\mathrm{n}^{\circ} 139$.

QUTB Jamâl, 1413 H., Al-Azhar fî-l-Busna wa-l-Harsak, (séries Majallatal-Azhar).

AL-SEMARI Fahd Ben Abdallah, 1992, Al-'amalal-islâmî fì urubba al-sharqiyya : al-tahaddiyat wa-lmustaqbal (L'œuvre islamique en Europe de l'est : défis et avenir), Université Ibn Sa'ûd.

SIBONY Daniel, Libération, 2 février 1991, cité par BENSLAMA F., «À propos de l'« Umma », Paysages après la bataille, contre la guerre des cultures, Paris, Esprit/Les Cahiers de l'Orient, p. 53-61.

\section{NOTES}

1. Voir Boltanski (1990), en référence à « l'action qui convient ».

2. L'occultation de cet aspect dans le débat est soulignée par Tarek Mitri (1993).

3. Dans les mêmes termes qu'un Bernard Henri-Lévy! 
4. Il y aurait sur le terrain quelques centaines seulement de combattants volontaires, le principal des efforts de soutien prenant la forme d'aides humanitaires assurées par une vingtaine d'organisations islamiques (al-Ahrâm, 10/08/1993).

INDEX

Mots-clés : altérité, Bosnie, Occident, Umma, islam, Europe

\section{AUTEUR}

IMAN FARAG

Cedej 